\title{
BMJ Open BREAST trial study protocol: evaluation of a non-invasive technique for breast reconstruction in a multicentre, randomised controlled trial
}

\author{
Sander S J Schop (D) , ${ }^{1}$ Juliette E Hommes, ${ }^{1}$ Todor K Krastev, ${ }^{1}$ Daniëlle Derks, ${ }^{2}$ \\ Mikko Larsen, ${ }^{3}$ HInne Rakhorst (D) , ${ }^{4,5}$ Ute Schmidbauer, ${ }^{6}$ Jan Maerten Smit, ${ }^{7}$ \\ Tik Tan, ${ }^{8,9}$ Kim Wehrens, ${ }^{8}$ Thijs de Wit, ${ }^{10}$ Rene R W J van der Hulst, ${ }^{1}$ \\ Andrzej A Piatkowski de Grzymala ${ }^{1}$
}

To cite: Schop SSJ, Hommes JE, Krastev TK, et al. BREAST trial study protocol: evaluation of a non-invasive technique for breast reconstruction in a multicentre, randomised controlled trial. BMJ Open 2021;11:e051413. doi:10.1136/ bmjopen-2021-051413

- Prepublication history and additional supplemental material for this paper are available online. To view these files, please visit the journal online (http://dx.doi.org/10.1136/ bmjopen-2021-051413)

Received 25 May 2021 Accepted 01 July 2021

\section{ABSTRACT}

Introduction Pioneers have shown that it is possible to reconstruct a full breast using just autologous fat harvested by liposuction or autologous fat transfer (AFT). This study describes the first multicentre randomised study protocol to thoroughly investigate the effectiveness of AFT to reconstruct full breasts following mastectomy procedures (primarily and delayed).

Methods and analysis This study is designed as a multicentre, randomised controlled clinical superiority trial with a 1:1 allocation ratio. A total of 196 patients (98 patients per treatment arm) are aimed to be included. Patients who wish to undergo breast reconstruction with either one of the two techniques are randomly allocated into the AFT group (intervention) or the tissue-expander/ prosthesis group (control). The primary outcome measure for the quality of life is measured by the validated BREAST-Q questionnaire.

Ethics and dissemination Approval for this study was obtained from the medical ethics committee of Maastricht University Medical Centre/Maastricht University; the trial has been registered at ClinicalTrials.gov. The results of this randomised controlled trial will be presented at scientific meetings as abstracts for poster or oral presentations and published in peer-reviewed journals.

Trial status Enrolment into the trial has started in October 2015. Data collection and data analysis are expected to be completed in December 2021.

Trial registration number NCT02339779.

\section{INTRODUCTION}

Breast cancer is a worldwide common malignancy for women. In the Netherlands, breast cancer is the most prevalent type of cancer with $29.5 \%$ of all malignancies in women. About one in eight women in the Netherlands will develop invasive breast cancer over the course of a lifetime. In our country in 2013, 14326 women were affected with invasive breast cancer, along with 2491 new cases of non-invasive (in situ) breast cancer. ${ }^{1}$ Treatment of breast cancer consists of surgery and

\section{Strengths and limitations of this study}

- The design is randomised and multicentred, which results in a higher external validity for it creates more representative and more comparable groups according to both known and unknown factors.

- This study is the first trial in which efficacy of autologous fat transfer (AFT) for full breast reconstruction is evaluated.

- Limited clinical equipoise for both treatment groups can introduce selection bias.

- There was no blinding performed in this study.

- Surgeons have years of experience in breast reconstruction, yet AFT is a new technique; thus, the final touch in the experimental arm may be impacted by a learning curve element.

might be accompanied by chemotherapy, radiation, hormonal therapy and immunotherapy. ${ }^{2}$ Depending on the type and tumour grade, patients are advised to undergo either mastectomy or breast-conserving surgery.

After the breast cancer treatment, many patients perceive problems in their perception of body image, sexuality and femininity and develop signs of depression and anxiety. ${ }^{34}$ A growing number of patients with breast cancer therefore choose to have the breast(s) reconstructed. Numerous studies have documented the significant improvement in self-confidence, body image, femininity, sexuality and overall mental health following breast reconstruction. ${ }^{5-7}$ Breast reconstruction thus aims to restore the quality of life (QoL) of patients with breast cancer by creating a breast that appears as natural as possible. In the Netherlands, approximately $20 \%$ of patients with breast cancer choose to undergo breast reconstruction. With the 
advancement and experience of surgical techniques, this number is expected to increase.

Current breast reconstruction techniques include alloplastic techniques using implant(s) or autologous techniques using a variety of flaps. ${ }^{8}$ Although surgeons strive for perfection, all current techniques have considerable disadvantages such as the need for revision surgery when using foreign body materials due to device problems or capsular contraction, or the fact that autologous tissue uses a donor site and being considerably invasive. This study aimed to investigate whether a third technique is effective as a breast reconstruction technique combining the advantages of autologous tissue while being minimally invasive: autologous fat transfer (AFT).

\section{Comparing current breast reconstruction methods}

While the advantage of using implants is that the technique is not as invasive as autologous tissue techniques, there are also disadvantages. One of these disadvantages with impact for the patient is that, over time, through the degradation of the silicone bladder, all implants will eventually leak. Other disadvantages include encapsulation that causes the breasts to gradually harden and change shape, which might also facilitate implant rupture. Some patients perceive problems due to the change in shape and consistency of their breast such as pain. Therefore, patients choosing breast reconstruction using implants are informed that implant exchanges may be necessary over the course of a lifetime.

Early complications inherent to the use of tissue expanders and implants include infections, haematomas and mastectomy skin necrosis. Late complications include capsular contractures and implant dislocation with resultant asymmetry, deflation and rippling. Implant extrusion can occur early because of skin necrosis or late as a result of fold-induced pressure points on the skin or incision line. Compared with free flaps, the advantages of breast reconstruction using implants are less invasive procedure, less visible scarring, shorter operation and hospitalisation times. Excellent cosmetic results are obtained with bilateral reconstructions. ${ }^{9-11}$

Using the patient's own tissue has some distinct advantages. It improves the natural feeling of the self after mastectomy compared with a synthetic implant. The use of soft tissue improves the ability to mimic natural ptosis in the reconstructed breast. This is hard to manage with unilateral implant reconstruction, as an implant will always feel firmer and will evolve differently over time than normal breast tissue. Also, breast size will vary with weight of the patient and with age, which will not be the case for the reconstructed breast.

For some women, an attractive benefit of using autologous tissue is the concomitant abdominoplasty when the abdominal skin and fat tissue are used as the donor area. The disadvantage is the relatively small but catastrophic possibility to lose a complete flap through necrosis resulting in additional surgery. With experience and appropriate selection of patients (low body mass index
(BMI), non-smokers, no history of thrombosis) complete flap loss rates of $0.5 \%$ are achievable. ${ }^{8}$ The disadvantages of autologous tissue using free flaps include longer operation and hospitalisation times, donor site morbidity (abdominal wall morbidity and hypertrophic and visual scarring), vascular flap complications (fat necrosis, partial flap necrosis and total flap failure) and general surgical complications (infection, bleeding, seroma and wound dehiscence). ${ }^{12} 13$

\section{Autologous fat transfer}

Lipofilling or AFT is a surgical technique in which fat is being transferred to the breast. Liposuction, on which this technique is based upon, is widely applied and is even characteristic for aesthetic surgery to reduce the fat layer in the abdomen, buttocks and hip region. In the face, it is widely applied to reduce the effects of ageing and is known as lipofilling. For reconstruction purposes, AFT is also a successful method to restore congenital or iatrogenic deformities in the face, thorax and extremities. Less commonly applied is AFT to the breast.

Over the past years, AFT has gained attention in breast reconstructive surgery. ${ }^{14-16}$ So far, AFT is mainly used for correction of breast deformities in partial breast reconstruction. Macromastia, tuberous breasts, Poland syndrome (it is an uncommon birth defect characterised by underdevelopment or absence of the chest muscle on one side of the body), postaugmentation deformity, postlumpectomy deformity, postmastectomy deformity, sequelae of postradiotherapy, secondary reconstruction after flap or implant reconstruction and nipple reconstruction. ${ }^{17-20}$ Recently, some case series have shown the possibility of reconstruction of a breast after mastectomy using AFT. ${ }^{19} 21-23$

The advantages of AFT are biocompatibility, diversity, natural appearance, and low donor site morbidity. ${ }^{22}$ These characteristics make AFT a promising breast reconstruction technique. However, until now, no randomised controlled study has addressed the effectiveness of AFT in (breast cancer) patients. ${ }^{24}$ Evidence relies on retrospective studies, case series or single-centre small cohort studies with a short follow-up. In these studies, no standardised techniques described the graft harvesting, preparation or injection. Some studies treat the transferred fat with stem cells or with growth factors to stimulate the survival of adipocytes (enriched lipofilling). ${ }^{24-27}$ Unfortunately, very little studies have assessed patientreported outcome measures. ${ }^{28}$ Thomson et $a l^{28}$ emphasise the importance to test the patient-reported outcomes in patients with breast cancer as the impact on their lives is rather large on psychosocial, physical and sexual aspects, including self-image and self-confidence. ${ }^{3}{ }^{4}$ To overcome the lack of strong methodology to study the effectiveness of $\mathrm{AFT}^{29}$ this study will research whether AFT is an effective new breast reconstruction method using a randomised controlled study. This study applies a variety of standardised outcome measures, including patient-reported outcomes (primary outcome measure) 
and volume and skin quality and (aesthetic) appearance as secondary outcome measures.

\section{METHODS AND ANALYSIS \\ Objective}

The primary objective was to determine if AFT is an effective new method compared with a two-staged implantbased breast reconstruction, the current gold standard. As the aim of breast reconstruction is to improve the patients' QoL the primary objective was to study whether the QoL of patients who undergo AFT is higher than patients in the control group receiving implants.

\section{Study design}

A multicentre randomised controlled clinical trial is designed. This study is carried out in seven hospitals in the Netherlands, of which two are university medical centres and five (high volume) regional hospitals. The recruitment process is illustrated in figure 1.

After inclusion and informed consent (see online supplemental material for model as provided by the Central Committee on Research Involving Human Subjects), patients are randomly allocated into the intervention group, sequential AFT with pre-expansion, or the control group with tissue-expander/prosthesis breast

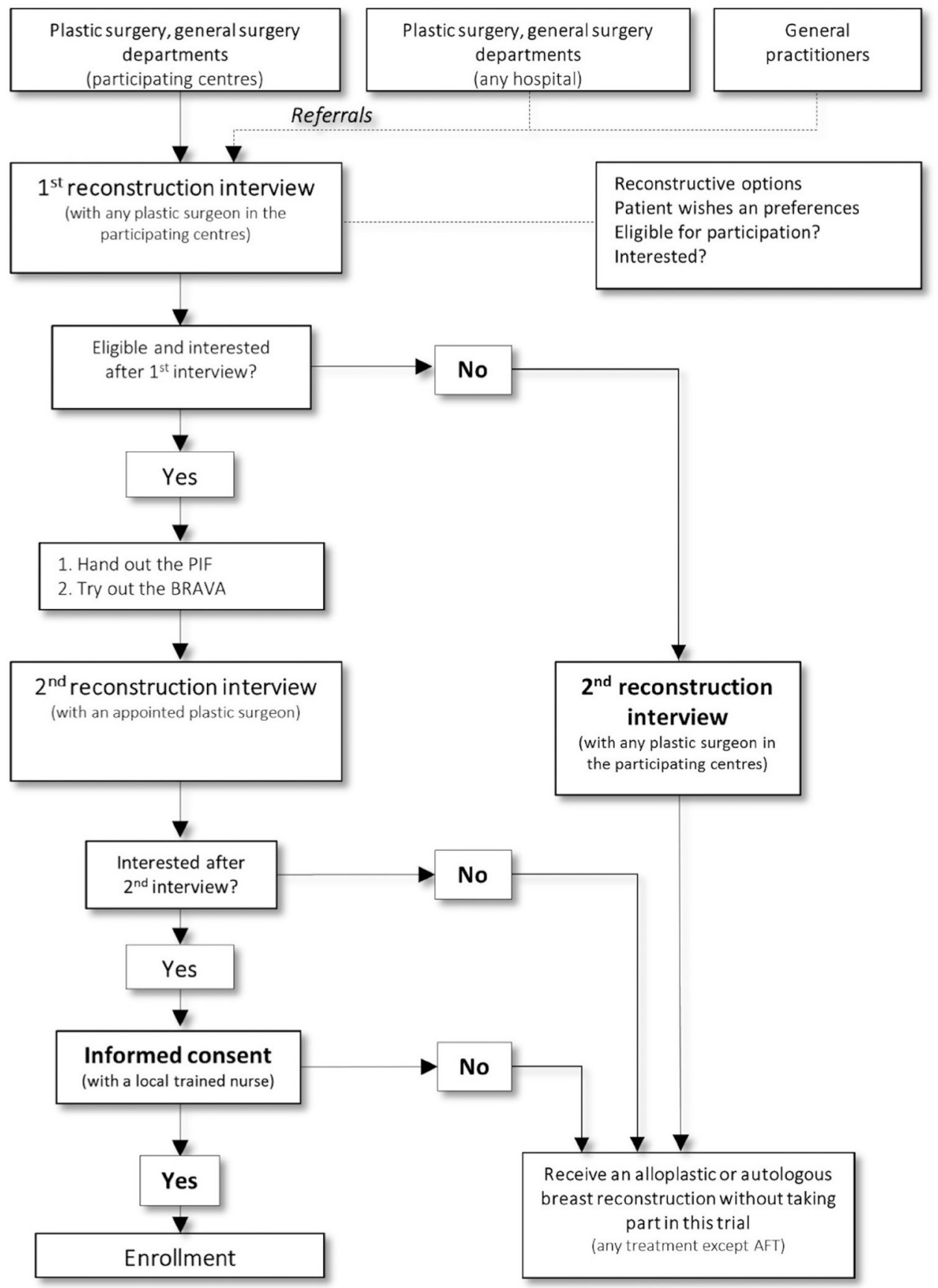

Figure 1 Overview of the recruitment process. AFT, autologous fat transfer; BRAVA, BRA like VAcuum-based external tissue expander; PIF, Patient information form. 


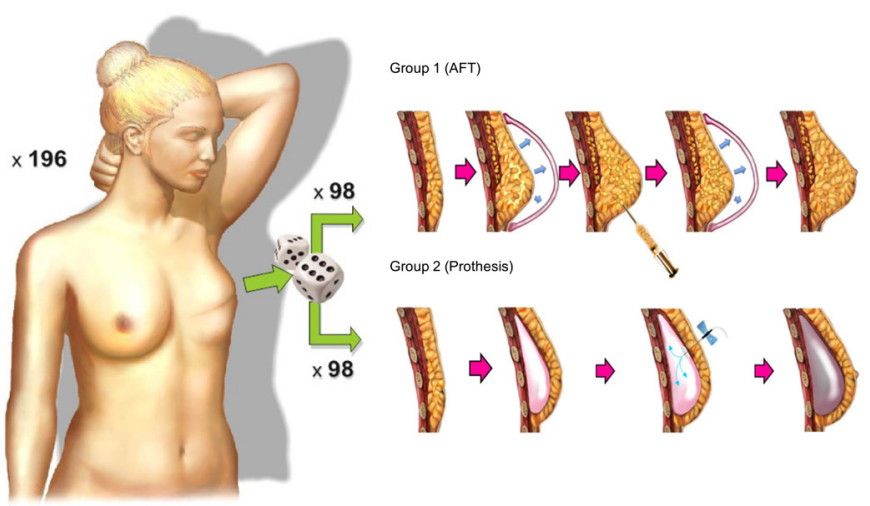

Figure 2 Trial design. Patients are randomly allocated to one of the two study groups - the AFT group or the prosthesis group (credits to TKK). AFT, autologous fat transfer.

reconstruction, as shown in figure 2 . This will be done by a 1:1 allocation as per a computer-generated randomisation schedule stratified by site using block randomisation. This procedure will take place 1 day before the patient undergoes surgery. She will also then be informed about the allocated treatment arm by telephone.

\section{Participants}

A total of 196 patients is aimed to be included in the BREAST trial. A patient can be referred to one of the participating centres by her treating surgeon or her general practitioner. The study team has actively been informing breast surgeons, as well as plastic surgeons about the study at national meetings. Also, the National Breast Cancer Patient Association refers to this research online. The study is open to women with breast cancer who are scheduled to undergo a mastectomy, women who wish to undergo prophylactic mastectomy because of an increased risk of developing breast cancer, as well as women who have already undergone a mastectomy in the past. Patients who have undergone a breast reconstruction before may participate, for instance, after a failed autologous tissue transfer (eg, Deep Inferior Epigastric Perforator - flap (DIEP)) or when a patient is in need of capsulectomy and replacement of implants after previous breast reconstruction, are also eligible for inclusion.

\section{Sample size calculation}

A power calculation was performed with QoL as the primary outcome measure. Multiple response variables are being assessed, with the average score of QoL measured by three scales of the BREAST-Q questionnaire as the primary outcome measure. ${ }^{3031}$ Sample size was estimated with the 'Pwr' package of the R software ${ }^{32}$ using the 'Pwr.t.test' function. Assuming a clinically relevant change in QoL of half an $\mathrm{SD},{ }^{33}$ a group size of 85 per arm is required for a two-tailed independent-samples t-test to detect a clinically relevant change with $90 \%$ power, effect size 0.50 Cohen and a significance level $(\alpha)$ of $0.05 .{ }^{34}$ To compensate for an estimated drop-out rate of $15 \%$, this study requires at least 98 patients per treatment arm.
Box 1 Inclusion and exclusion criteria

Inclusion criteria

- Female gender.

- Age of 18 years and older.

- History or in candidate for a mastectomy in the near future.

- Patients undergoing prophylactic mastectomy.

- Patients' choice to undergo a breast reconstruction.

- Wanting to participate in this study.

- Patient is able to wear the BRAVA (BRA like VAcuum-based external tissue expander) device.

\section{Exclusion criteria}

- Active smoker or a history of smoking 4 weeks prior to surgery.

- Current substance abuse.

- History of lidocaine allergy.

- Four weeks or less after chemotherapy.

- History of radiation therapy in the breast region.

- Oncological treatment includes radiotherapy after mastectomy.

- Kidney disease.

- Steroid dependent (daily or weekly).

- Immune-suppressed or immune-compromised disease.

- Uncontrolled diabetes.

$>$ body mass index of $>30$.

- Large breast size (ie, larger than cup C), unless the patient prefers reduction of the contralateral side towards cup C.

- Extracapsular silicone leaking from the encapsulated implant from a previous breast reconstruction.

\section{Inclusion and exclusion criteria}

In box 1 , an overview of the inclusion and exclusion criteria is shown.

\section{Interventional surgical technique: AFT}

Pre-expansion

The treatment for the patients randomised to the intervention is illustrated in figure 3. Patients are asked to wear the BRAVA (BRA like VAcuum-based external tissue

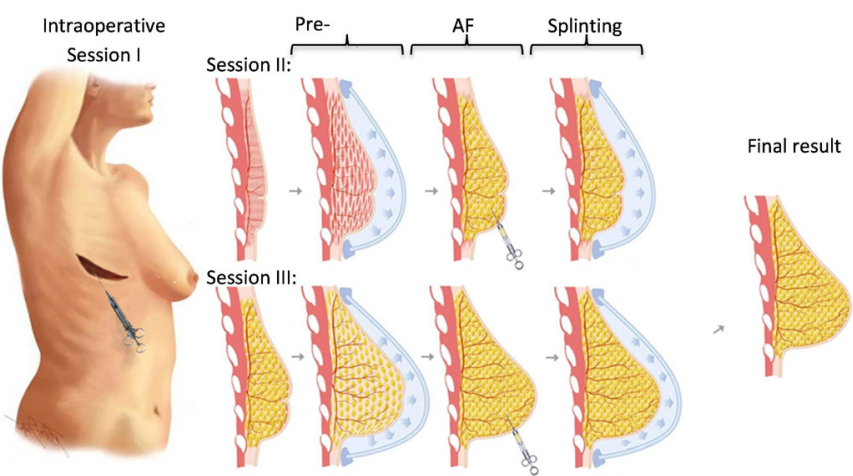

Figure 3 Sequence of the treatment for patients in the experimental group. AFT will occur in three steps: preexpansion for 4 weeks using the BRAVA device, AFT and postoperative splint using the BRAVA for 2 more weeks. The full session can be repeated to reach the intended volume (credits to TKK). AF, autologous fat; AFT, autologous fat transfer; BRAVA, BRA like VAcuum-based external tissue expander. 
expander) external breast tissue expander for a minimum of 10 hours/day during 4 weeks prior to the surgery date.

\section{Surgery}

The outcome of the surgical treatment is dependent on the surgeons' skills and experience. Surgeons were approached to participate in this study due to their previous experience. One 'hands-on' training session with the previously mentioned surgeons will strive for one general technique for harvesting and transferring the fat grafts.

The AFT surgery is performed under general anaesthesia.

\section{Fat grafting}

The technique used for harvesting fat cells and lipofilling is already applied for other purposes in hospitals, described by Coleman and Saboeiro. ${ }^{22}$

Harvesting: First, small 2-3 mm incisions are made in the area where fat is to be harvested. Consequently, a solution of saline, lidocaine with epinephrine is infiltrated in the fat grafting area, following protocol (infiltration fluid before liposuction: $500 \mathrm{~mL} \mathrm{NaCl}, 0.5 \mathrm{~mL}$ epinephrine $(0.5 \mathrm{mg} / \mathrm{mL}), 2.5 \mathrm{~mL}$ lidocaine $(1 \%))$. Then, fat is harvested using a $10 \mathrm{~mL}$ syringe attached to a two-hole blunt harvesting cannula. When the syringe is full, the aspirated fat is transferred to the Puregraft collection bag.

Processing: The Puregraft filtration system is used to collect and purify the lipoaspirate by washing out contaminants such as blood, infiltration fluid of oil from ruptured adipocytes. When this is achieved, the semiliquid lipoaspirate is transferred to $3-10 \mathrm{~mL}$ syringes.

Fat injection: The principal issue for survival emphasises the need to provide the grafts with sufficient access to nutrition and respiration to survive until neovascularisation takes place. Approximately $60 \%$ of the transferred fat cells that are more than $1 \mathrm{~mm}$ from a source of nutrition and respiration will die. ${ }^{22}$ Therefore, the fat graft is injected in microdroplets and aliquots, fanning the fat graft in different planes (prepectoral, intrapectoral and retropectoral), and into the deep and superficial dermis of the breast to maximise the chance of survival. ${ }^{35}$ Shaping of the breast is consequently accomplished by layering the fat into different levels until the desired contour is achieved.

\section{Post-AFT procedure}

After surgery, the patient continues to wear the BRAVA device for 2 weeks. It serves the purpose of a splint protecting and immobilising the grafted fat while providing negative pressure that stimulates neovascularisation. The fat graft body surface is supported by pressure compression garment for 2-4 weeks.

\section{Repetition of a breast reconstruction session}

Depending on the shape of the contralateral breast or prior breast size, on average, two more sessions might be necessary. In general, the session will be repeated up to a maximum of five sessions until an acceptable volume and

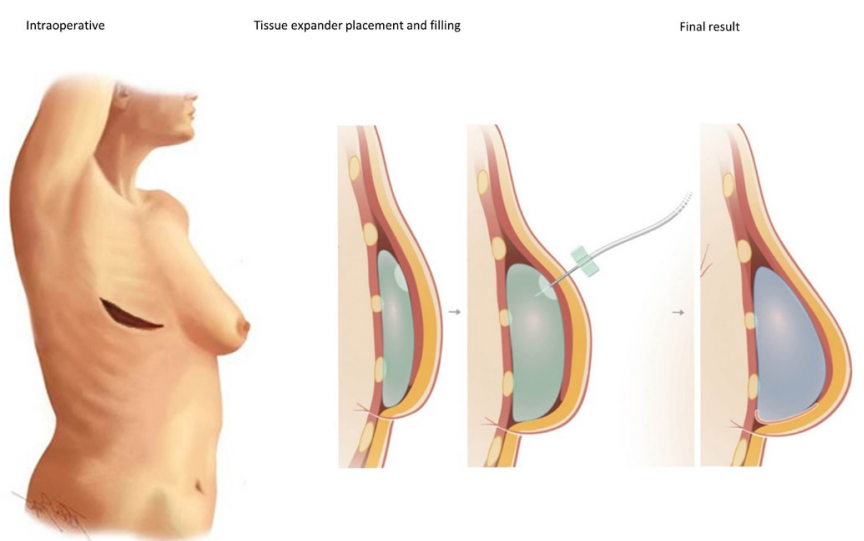

Figure 4 Sequence of breast reconstruction for the patients allocated to the control group: (1) schematic representation of a subpectoral tissue expander after mastectomy, (2) filling of the tissue expander in the outpatient clinic and (3) final result after exchange of the tissue expander with the definitive implant (credits to TKK).

shape are achieved. Depending on the patients' wish, the second session can start after a minimum of 4 weeks after the last session has ended, which means that the surgery will be at least 10 weeks apart.

\section{Alloplastic breast reconstruction using implants}

The patients randomised to the control group will undergo a breast implant based on a certain shape and volume judged by the treating plastic surgeon and the desired final breast volume, as is illustrated in figure 4 .

\section{Mastectomy and first-phase breast reconstruction First surgery}

After the oncological surgeon has finished the mastectomy, a pocket for the tissue expander is created beneath the major pectoralis muscle. After haemostasis is reached and local antiseptic measures are taken, the tissue expander with a maximum volume of $10 \mathrm{cc}$ injected saline will be placed in the pocket. The edges of the major pectoralis and serratus anterior muscle will be sutured. Based on the surgeons' practice, a drain is inserted through a separate stab incision, tunnelled through a layer of subcutaneous tissue. The skin is closed in a standard layered fashion.

\section{Expansion}

Within 14 days after the placement of the tissue expander, the external sutures will be removed and the tissue expander can be filled with 50-100 cc per outpatient clinical visit until the desired volume is reached. Consequent filling sessions can be planned at least 3 weeks after the last expansion took place. In the following surgery, the final implant will replace the tissue expander.

\section{Second surgery}

The mastectomy scar is excised and the muscular pocket is opened. The encapsulated implant is removed (with capsule). After haemostasis is reached and local antiseptic measures are taken, the definite implant is inserted in the muscular pocket. The edges of the major pectoralis 


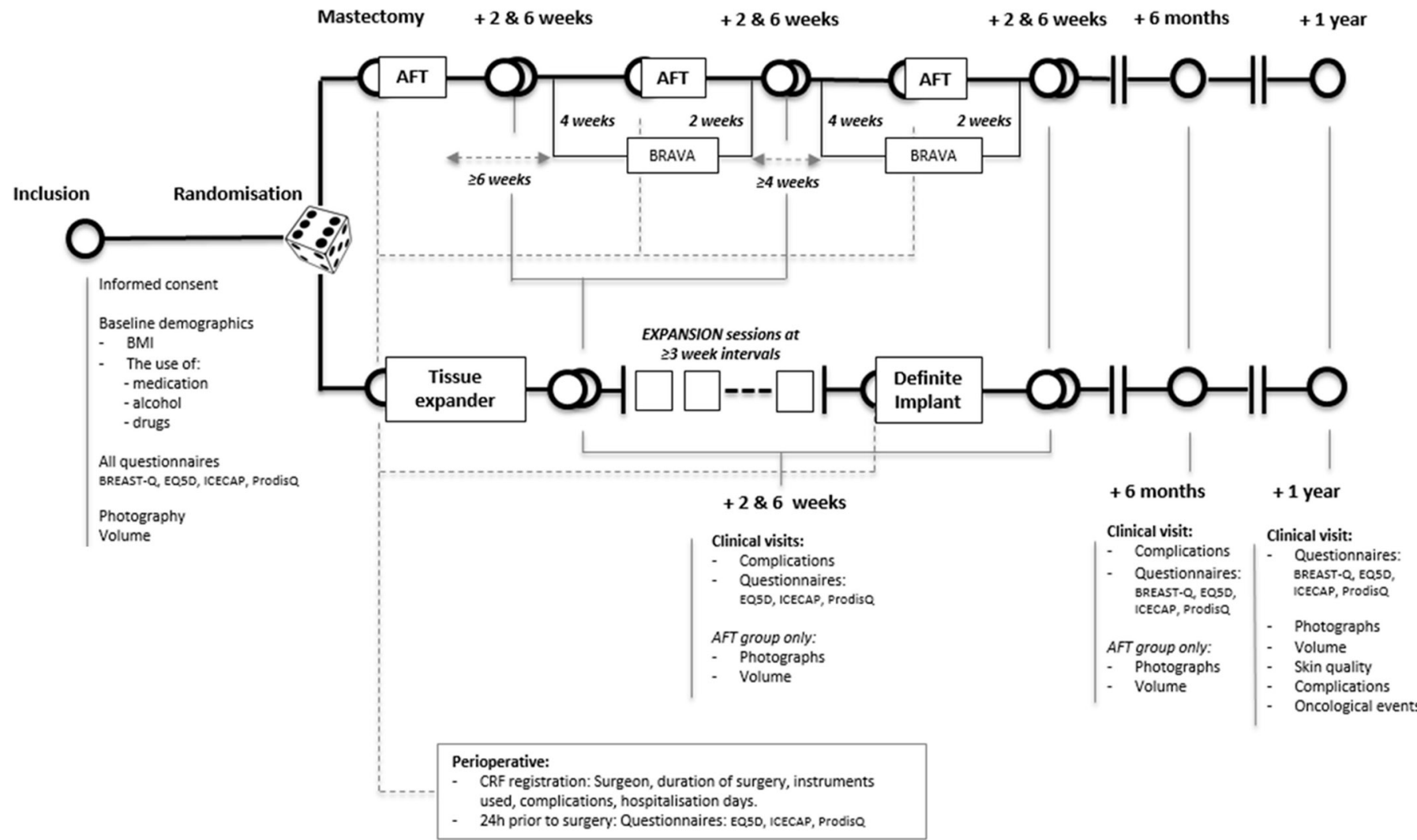

Figure 5 Schematic overview for follow-up of outcome measures. AFT, autologous fat transfer; BMI, body mass index; ICECAP-A, Investigating Choice Experiences for Capabilities in Adults.

and serratus anterior muscle will be sutured. Based on the surgeons' practice, a drain is inserted through a separate stab incision, tunnelled through a layer of subcutaneous tissue. The skin is closed in a standard layered fashion.

\section{Encapsulated implant that needs replacement}

In case a patient has undergone a mastectomy and reconstruction with implants in the past that need replacement due to severely encapsulated or ruptured implants, a similar surgery takes place as described in the Second surgery section.

\section{Outcome measures}

The primary endpoint is QOL, as breast reconstruction intends to enhance this. This parameter will be assessed using three subscales of the BREAST-Q: emotional, sexual and physical well-being. Next to QoL, the quality of the breast reconstruction is measured by the volume and shape over time (3D photography or MRI), patient satisfaction (BREAST-Q questionnaire) and aesthetic judgement (panel rating 3D preoperative and postoperative photos). See figure 5 for the time points applied in the study. Also, complications during the treatment and follow-up period will be registered and compared between the intervention and control groups.

Besides, oncological follow-up will be monitored for 5 years, using imaging reports and chart analysis to determine the development of local recurrence or distant metastatic disease related to the original breast tumour.
Furthermore, a cost-effectiveness analysis will be performed. This will be done by gathering data in the electronic Case Report Form (eCRF) from all patients included in the multicentre trial for the total duration of follow-up with regard to resource use. Resource data are associated with rehabilitation, surgery (surgeon, surgery duration instruments used, volume of fat transferred and/or implants used), hospitalisation, (extra) visits to clinics and treatment of complications. The costs will be calculated per patient and will be compared between the control and intervention groups. The measures of effectiveness will be general wellbeing weighted survival and generic health-related quality of life weighted survival (eg, quality-adjusted life years (QALYs)). ProdisQ is used to gather information regarding (loss) of productivity in labour or non-paid labour. In case of a complication, ProdisQ Investigating Choice Experiences for Capabilities in Adults (ICECAP-A) and EQ5D-5L will be completed at the time of complication and when the complication subsides to understand the impact of the complication on the health-related quality of life.

\section{Data analysis}

Per patient preoperative and postoperative emotional, physical and sexual well-being will be calculated using the BREAST-Q subscale. A two-tailed independent sample t-test will determine the difference in quality of life between the intervention (AFT) and control (implant) groups at baseline and 1 year postoperatively. 
To investigate the development of the quality of life over time between the intervention and control groups, a multilevel regression analysis using time as the withinsubjects factor, and the study group as the betweensubjects factor.

Confounders such as heterogeneity in the patient demographics between centres, type of mastectomy treatment (skin-sparing, nipple sparing and regular mastectomy), differences between patients undergoing primary and secondary breast reconstruction and surgeon-related differences in the outcome could still exist. Correlational analyses will search for these possible confounding factors, while regression analysis can take into account possible confounding factors.

Problems with patient compliance and loss to follow-up inevitably lead to incomplete data. To reduce the drop-out or loss-to follow-up, we try to inform patients thoroughly what happens when participating in this trial, including the possibility to experience the BRAVA device. The oncological follow-up can be done in any hospital. To compensate for loss to follow-up, an overcorrection of $15 \%$ has been applied in the sample size estimation during the design stage of this trial. In the analytical stage, missings will be identified and classified accordingly as 'missings completely at random', 'missings at random' and 'missings not at random'. Missing data will be then handled appropriately using validated methods for dealing with missings (eg, multiple imputation, maximum likelihood estimation approach or other).

\section{Economic evaluation}

The economic analysis will be conducted from a healthcare perspective with a time horizon of 1 year. Healthcare costs will be calculated by multiplying resource use with cost prices selected based on using the 'Handleiding Kostenonderzoek' of the National Health Institute. ${ }^{36}$ The total costs per patient will be calculated. The scores of the ProdisQ questionnaires will be used to calculate the lost productivity per patient over time. The scores on the ICECAP-A and the EQ5D-5L will be used to calculate population-based utility weights using the accompanying tariffs. ${ }^{36}$ These population utility weights will be used to calculate two QALYs: one capturing well-being weighted survival (using ICECAP-A scores) and one capturing health-related quality of life weighted survival (EQ5D-5L). Subsequently, two incremental cost-effectiveness ratios will be determined by dividing the between-group difference in costs by the between-group difference in QALYs. Uncertainty surrounding the ratios will be assessed using non-parametric bootstrapping. Acceptability curves will be drawn to visualise the probability that AFT is costeffective compared with two-staged breast reconstruction using implants.

\section{Patient and public involvement}

Patients were not involved in the development of the research question, study design or recruitment into the study.

\section{Ethics and dissemination}

This study will be conducted according to the principles of the Declaration of Helsinki (64th WMA General Assembly, Fortaleza, Brazil, October 2013) and in accordance with the Medical Research Involving Human Subjects Act.

The described study was approved by the medical ethics committee (MEC) of Maastricht University Medical Centre/Maastricht University. All amendments made to the protocol will be first proposed to the MEC. After approval, they will be communicated to all involved parties.

\section{Informed consent}

All patients will have to sign an informed consent form before participating in the trial.

\section{Data validation and management}

All data will be registered and analysed coded. Patient data are accessed only after informed consent is provided and by the investigators only. All subjects will be given insight into the (individual) data of this study by the investigators.

\section{Data monitoring}

An independent data monitoring committee consisting of certified and qualified personnel from the Clinical Trial Centre Maastricht will monitor compliance with the study procedures as well as quality of the collected data, following their protocol as requested by the board.

\section{Harms}

Adverse events (AEs) are defined as any undesirable experience occurring to a subject during the study, whether or not considered related to the trial procedure. AEs related to the BRAVA-AFT operation or standard therapy that have a possible impact on the reconstruction and reported spontaneously by the subject or observed by the investigator or his staff will be recorded directly in the eCRF. The research team will report the serious adverse event (SAEs) through the web portal ToetsingOnline to the accredited Medical Ethics Committee (METC) that approved the protocol, within 7 days of first knowledge for SAEs that result in death or are life-threatening followed by a maximum period of 8 days to complete the initial preliminary report. All other SAEs will be reported within a period of a maximum 15 days after the research team has first knowledge of the SAEs.

\section{Dissemination}

The results of this trial will be submitted for publication to international high-impact, scientific journals, regardless of the outcome of this study, as well as presented at international conferences. Publications will be ascribed in accordance with the International Committee of Medical Journal Editors guideline.

\section{Confidentiality}

All reports, data collection, process and administrative forms are identified by a coded identification (ID) 
number only to maintain participant confidentiality. All records that contain names or other personal identifiers, such as locator forms and informed consent forms, will be stored separately from study records identified by code number. All local databases will be secured with passwordprotected access systems. Forms, lists, logbooks, appointment books and any other listings that link participant ID numbers to other identifying information will be stored in a separate, locked file in an area with limited access.

\section{Trial status}

Enrolment into the trial has started in October 2015. Data collection and data analysis are expected to be completed in December 2022.

\section{Author affiliations}

${ }^{1}$ Plastic, Reconstructive, and Hand Surgery, Maastricht University Medical Centre+, Maastricht, The Netherlands

${ }^{2}$ Plastic, Reconstructive, and Hand Surgery, Alexander Monro Breast Cancer Hospital, Bilthoven, The Netherlands

${ }^{3}$ Plastic, Reconstructive, and Hand Surgery, Launceston General Hospital, Launceston, Tasmania, Australia

${ }^{4}$ Plastic, Reconstructive and Hand Surgery, Medisch Spectrum Twente, Enschede, The Netherlands

${ }^{5}$ Plastic, Reconstructive and Hand Surgery, Ziekenhuisgroep Twente, Almelo, The Netherlands

${ }^{6}$ Plastic, Reconstructive, and Hand Surgery, Hospital Group Twente Hengelo, Hengelo, The Netherlands

${ }^{7}$ Plastic, Reconstructive, and Hand Surgery, VU University Medical Centre Amsterdam, Amsterdam, The Netherlands

${ }^{8}$ Plastic, Reconstructive, and Hand Surgery, Medical Centre Haaglanden, Den Haag, The Netherlands

${ }^{9}$ Plastic Surgery, HMC The Hague, Barendrecht, The Netherlands

${ }^{10}$ Plastic, Reconstructive, and Hand Surgery, Amphia Hospital, Breda, The Netherlands

Acknowledgements We thank all patients, investigators, patient advisors and institutions involved in this study, such as the Dutch Health Institute, the Dutch Breast Cancer Association and Health Insurance Alliance Netherlands.

Contributors AAPdG, RRWJvdH, TKK and JEH initially conceived the study and initiated the study design. SSJS, JEH, TKK, DD, ML, HR, US, JMS, TT, KW, TdW, RRWJvdH and AAPdG contributed to the eventual study protocol and are responsible for execution accordingly. SSJS, RRWJvdH, JEH and AAPdG completed this version of the study design and protocol. All authors contributed to the refinement of the study protocol and approved the final version.

Funding This work was funded by ZonMw; The Netherlands Organisation for Health Research and Development (grant number 80-83700-98-15505).

Disclaimer The funder will not have any authority over any of the studyrelated activities, consisting of data collection, data management, data analysis, interpretation of results, writing the report nor submission for publication.

Competing interests None declared.

Patient consent for publication Not required.

Provenance and peer review Not commissioned; externally peer reviewed.

Supplemental material This content has been supplied by the author(s). It has not been vetted by BMJ Publishing Group Limited (BMJ) and may not have been peer-reviewed. Any opinions or recommendations discussed are solely those of the author(s) and are not endorsed by BMJ. BMJ disclaims all liability and responsibility arising from any reliance placed on the content. Where the content includes any translated material, BMJ does not warrant the accuracy and reliability of the translations (including but not limited to local regulations, clinical guidelines, terminology, drug names and drug dosages), and is not responsible for any error and/or omissions arising from translation and adaptation or otherwise.

Open access This is an open access article distributed in accordance with the Creative Commons Attribution Non Commercial (CC BY-NC 4.0) license, which permits others to distribute, remix, adapt, build upon this work non-commercially, and license their derivative works on different terms, provided the original work is properly cited, appropriate credit is given, any changes made indicated, and the use is non-commercial. See: http://creativecommons.org/licenses/by-nc/4.0/.

\section{ORCID iDs}

Sander S J Schop http://orcid.org/0000-0001-5187-4144

HInne Rakhorst http://orcid.org/0000-0002-1663-6092

\section{REFERENCES}

1 Kankerregistratie, May, 2014. Available: Borstkanker (iknl.nl)

2 NABON. Mammacarcinoom Richtlijn versie 2.0: KNL Integraa kankercentrum Nederland, 2012. Oncoline

3 Bloom JR, Stewart SL, Oakley-Girvan I, et al. Quality of life of younger breast cancer survivors: persistence of problems and sense of well-being. Psychooncology 2012;21:655-65.

4 Høyer M, Johansson B, Nordin K, et al. Health-related quality of life among women with breast cancer - a population-based study. Acta Oncol 2011;50:1015-26.

5 Eltahir Y, Werners LLCH, Dreise MM, et al. Quality-Of-Life outcomes between mastectomy alone and breast reconstruction: comparison of patient-reported BREAST-Q and other health-related quality-of-life measures. Plast Reconstr Surg 2013;132:201e-9.

6 Zhong T, McCarthy C, Min S, et al. Patient satisfaction and healthrelated quality of life after autologous tissue breast reconstruction. Cancer 2012;118:1701-9.

7 Macadam SA, Ho AL, Lennox PA, et al. Patient-Reported satisfaction and health-related quality of life following breast reconstruction: a comparison of shaped cohesive gel and round cohesive gel implant recipients. Plast Reconstr Surg 2013;131:431-41.

8 Sigurdson L, Lalonde DH. Breast reconstruction. Plast Reconstr Surg 2008;121:1-12.

9 Cordeiro PG, McCarthy CM. A single surgeon's 12-year experience with tissue expander/implant breast reconstruction: Part I. A prospective analysis of early complications. Plast Reconstr Surg 2006;118:825-31.

10 Cordeiro PG, McCarthy CM. A single surgeon's 12-year experience with tissue expander/implant breast reconstruction: Part II. An analysis of long-term complications, aesthetic outcomes, and patient satisfaction. Plast Reconstr Surg 2006;118:832-9.

11 Handel N, Cordray T, Gutierrez J, et al. A long-term study of outcomes, complications, and patient satisfaction with breast implants. Plast Reconstr Surg 2006;117:757-67.

12 Hofer SOP, Damen THC, Mureau MAM, et al. A critical review of perioperative complications in 175 free deep inferior epigastric perforator flap breast reconstructions. Ann Plast Surg 2007:59:137-42.

13 Gill PS, Hunt JP, Guerra AB, et al. A 10-year retrospective review of 758 DIEP flaps for breast reconstruction. Plast Reconstr Surg 2004;113:1153-60.

14 Agha RA, Goodacre T, Orgill DP. Use of autologous fat grafting for reconstruction postmastectomy and breast conserving surgery: a systematic review protocol. BMJ Open 2013;3:e003709:10.

15 Critchley AC, Thompson AM, Chan HY, et al. Current controversies in breast cancer surgery. Clin Oncol 2013;25:101-8.

16 Kling RE, Mehrara BJ, Pusic AL, et al. Trends in autologous fat grafting to the breast: a national survey of the american society of plastic surgeons. Plast Reconstr Surg 2013;132:35-46.

17 Gutowski KA, Force A, ASPS Fat Graft Task Force. Current applications and safety of autologous fat grafts: a report of the ASPS fat graft Task force. Plast Reconstr Surg 2009;124:272-80.

18 Sarfati I, Ihrai T, Kaufman G, et al. Adipose-Tissue grafting to the post-mastectomy irradiated chest wall: preparing the ground for implant reconstruction. J Plast Reconstr Aesthet Surg 2011;64:1161-6.

19 Panettiere P, Accorsi D, Marchetti L, et al. Large-breast reconstruction using fat graft only after prosthetic reconstruction failure. Aesthetic Plast Surg 2011;35:703-8.

20 Sinna R, Delay E, Garson S, et al. Breast fat grafting (lipomodelling) after extended latissimus dorsi flap breast reconstruction: a preliminary report of 200 consecutive cases. J Plast Reconstr Aesthet Surg 2010;63:1769-77.

21 Delay E, Garson S, Tousson G, et al. Fat injection to the breast: technique, results, and indications based on 880 procedures over 10 years. Aesthet Surg J 2009;29:360-76.

22 Coleman SR, Saboeiro AP. Fat grafting to the breast revisited: safety and efficacy. Plast Reconstr Surg 2007;119:775-85. 
23 Illouz YG, Sterodimas A. Autologous fat transplantation to the breast: a personal technique with 25 years of experience. Aesthetic Plast Surg 2009;33:706-15

24 Largo RD, Tchang LAH, Mele V, et al. Efficacy, safety and complications of autologous fat grafting to healthy breast tissue: a systematic review. J Plast Reconstr Aesthet Surg 2014;67:437-48.

25 Chan CW, McCulley SJ, Macmillan RD. Autologous fat transfer--a review of the literature with a focus on breast cancer surgery. J Plast Reconstr Aesthet Surg 2008;61:1438-48.

26 Rosing JH, Wong G, Wong MS, et al. Autologous fat grafting for primary breast augmentation: a systematic review. Aesthetic Plast Surg 2011:35:882-90.

27 Gir P, Brown SA, Oni G, et al. Fat grafting: evidence-based review on autologous fat harvesting, processing, reinjection, and storage. Plast Reconstr Surg 2012;130:249-58.

28 Thomson HJ, Winters ZE, Brandberg Y, et al. The early development phases of a European organisation for research and treatment of cancer (EORTC) module to assess patient reported outcomes (pros) in women undergoing breast reconstruction. Eur J Cancer 2013;49:1018-26.

29 Krastev TK, Jonasse Y, Kon M. Oncological safety of autologous lipoaspirate grafting in breast cancer patients: a systematic review. Ann Surg Oncol 2013;20:111-9.
30 Aguinis $\mathrm{H}$, Beaty JC, Boik RJ, et al. Effect size and power in assessing moderating effects of categorical variables using multiple regression: a 30-year review. J Appl Psychol 2005;90:94-107.

31 Faul F, Erdfelder E, Lang A-G, et al. G*Power 3: a flexible statistical power analysis program for the social, behavioral, and biomedical sciences. Behav Res Methods 2007;39:175-91.

$32 \mathrm{R}$ core team (2013). R: a language and environment for statistical computing. R foundation for statistical computing V, Austria. Available: http://www.R-project.org/

33 Norman GR, Sloan JA, Wyrwich KW. Interpretation of changes in health-related quality of life. Med Care 2003;41:582-92.

34 Aguinis H, Beaty JC, Boik RJ, et al. Effect size and power in assessing moderating effects of categorical variables using multiple regression: a 30-year review. J Appl Psychol 2005;90:94-107.

35 Khouri RK, Eisenmann-Klein M, Cardoso E, et al. Brava and autologous fat transfer is a safe and effective breast augmentation alternative: results of a 6-year, 81-patient, prospective multicenter study. Plast Reconstr Surg 2012;129:1173-85.

36 Zorginstituut Kostenhandleiding: Methodologie van kostenonderzoek en referentieprijzen voor economische evaluaties in de gezondheidszorg, 2015 